\title{
Biologia de Hylesia nigricans (Berg) (Lepidoptera, Saturniidae, Hemileucinae)
}

\author{
Alexandre Specht ${ }^{1}$; Aline C. Formentini ${ }^{1} \&$ Elio Corseuil ${ }^{2}$ \\ ${ }^{1}$ Laboratório de Biologia, Departamento de Ciências Exatas e da Natureza, Campus Universitário da Região dos Vinhedos, \\ Universidade de Caxias do Sul. Caixa Postal 32, 95700-000 Bento Gonçalves, Rio Grande do Sul, Brasil. \\ E-mail: aspecht@ucs.br, acformen@ucs.br \\ ${ }^{2}$ Laboratório de Entomologia, Faculdade de Biociências, Pontifícia Universidade Católica do Rio Grande do Sul. \\ Caixa postal 1429, 90619-900 Porto Alegre, Rio Grande do Sul, Brasil. E-mail: corseuil@pucrs.br
}

\begin{abstract}
Biology of Hylesia nigricans (Berg) (Lepidoptera, Saturniidae, Hemileucinae). The species of Hylesia Hübner, [1820] detach from other lepidopterous due to its urticant spines in larval (erucism) and adult (lepidopterism) stages. Hylesia nigricans (Berg, 1875) occurs from southeast of Brazil to Argentina and Uruguay, the species has been related to plague of many fruit crops and forest essences and they have also been associated with several lepidopterism cases since the beginning of last century. Due to its importance, this study aimed to detail the main biological aspects of this species with observations in the nature and in laboratory, they were also related behavioral aspects and host plants observed in field and referred in bibliography.

KEY WORDS. Biological parameters; caterpillars; erucism; life cycle; lepidopterism.

RESUMO. Biologia de Hylesia nigricans (Berg) (Lepidoptera, Saturniidae, Hemileucinae). As espécies de Hylesia Hübner, [1820] destacam-se entre os demais lepidópteros por apresentarem ação urticante tanto durante a fase larval (erucismo) quanto na fase adulta (lepidopterismo). Hylesia nigricans (Berg, 1875) cuja ocorrência é registrada do sudeste do Brasil até Argentina e Uruguai vem sendo relacionada como praga de diversas frutíferas cultivadas e essências florestais, também têm sido relacionada com diversos episódios de lepidopterismo desde o início do século passado. Tendo em vista a sua importância, este estudo objetivou detalhar os principais aspectos biológicos da espécie com observações na natureza e em laboratório, também foram relacionados aspectos comportamentais e plantas hospedeiras observadas no campo e referidas em bibliografia.

PALAVRAS CHAVE. Parâmetros biológicos; lagartas; erucismo; ciclo de vida; lepidopterismo.
\end{abstract}

Entre os lepidópteros de importância para o homem destacam-se alguns grupos cujos representantes apresentam estruturas com capacidade de produzir e de inocular substâncias de ação urticante ou hemolítica. No Brasil, merecem destaque as famílias Arctiidae, Limacodidae, Megalopygidae e Saturniidae. A maior parte dos acidentes ocorre em função do contato com as lagartas, entretanto, entre os representantes gênero Hylesia, além das lagartas apresentarem ação urticante, as fêmeas adultas possuem escamas abdominais modificadas em cerdas, utilizadas para recobrir e proteger suas posturas, que podem se desprender e causar dermatites (HADDAD \& CARDoso 2003).

O gênero Hylesia, exclusivamente neotropical, é composto por aproximadamente 110 espécies muito semelhantes entre si e de difícil identificação (Lemaire 2002).

Hylesia nigricans (Berg, 1875) que tem por sinônimo Hylesia fulviventris Berg, 1883, tem distribuição desde o Sudeste do Brasil (SiLva et al. 1968) até a Argentina (Berg 1875) e Uruguai (Bizzanko et al. 1957). Ocorre em grande número de exemplares sobre diversas árvores e frutíferas cultivadas, muitas vezes, de- vorando toda a folhagem (DRAUDT 1929). Devido ser muito comum, desde o início do século passado sua presença esteve relacionada com publicações sobre casos de dermatites envolvendo principalmente relatos de interesse médico (DALLAS 1926, 1933, Jörg 1933, 1935, 1969, CASALÁ et al. 1967, Arias 1981). Também existem trabalhos sobre alguns aspectos de biologia (MABILDE 1896, Bourquin 1944, LAMpe 1986), plantas hospedeiras (BiEZANKo et al. 1974) e de ocorrência Biezanko et al. 1957, 1978, Silva et al. 1968, Biezanko 1986, Corseuil et al. 2002).

Apesar da grande importância, tanto como desfolhadora de plantas como pela sua ação urticante durante a fase larval e adulta, não existem estudos detalhados sobre a biologia desta espécie. Assim, este trabalho teve por objetivo determinar os principais parâmetros biológicos de $H$. nigricans em laboratório.

\section{MATERIAL E MÉTODOS}

Este estudo foi realizado com observações em duas agregações de lagartas em campo (Fig. 1) e uma criação laboratorial sob temperatura de $25 \pm 2^{\circ} \mathrm{C}$, umidade relativa de $70 \pm 10 \%$ UR 
e fotofase de 14 horas. A criação foi iniciada a partir de três posturas coletadas sobre ramos de caneleira-preta - Nectandra megapotamica (Spreng.) Mez (Lauraceae), cujas folhas foram utilizadas para a alimentação das lagartas.

Os aspectos morfológicos referentes às fases de desenvolvimento foram avaliados efetuando-se mensurações com paquímetro digital e microscópio estereoscópico provido de ocular micrométrica com precisão de centésimo de milímetro e balança digital com precisão de centésimo de grama.

Fase de ovo - as posturas foram individualizadas em placas de petri com fundo forrado com papel filtro umedecido com água destilada, onde permaneceram até a eclosão das lagartas. Além das mensurações do maior comprimento e largura, avaliou-se o número de ovos por fêmea e também o percentual de eclosão.

Fase de lagarta - inicialmente 100 lagartas foram colocadas em recipientes plásticos com capacidade de $300 \mathrm{ml}$, para observações individuais; seus resultados deixaram de ser considerados porque somente quatro exemplares atingiram a fase adulta. Em função disso, as demais foram criadas em grupos provenientes de posturas com 88, 128, 156, 167 e 256 indivíduos, mantidas em gaiolas plásticas, cilíndricas de $19 \mathrm{~cm}$ de diâmetro por 23 de altura. A alimentação foi disponibilizada em ramos da planta hospedeira com extremidades introduzidas em Erlenmeier de $50 \mathrm{ml}$ com água destilada, para manter a turgescência; devido ao hábito gregário das lagartas e tecerem superfícies sedosas unindo as folhas para construção de um abrigo, foram mantidos os ramos utilizados por elas, renovando-se diariamente os destinados a alimentação.

Para a diferenciação dos instares, como referido em PARRA \& HADDAD (1989), organizou-se uma distribuição de freqüência das medidas das maiores larguras das cápsulas cefálicas recolhidas durante a criação. Ao final das medições, avaliou-se a razão de crescimento visando a sua adequação ao modelo linear de Dyar (1890).

Considerou-se como início do período de pré-pupa quando as lagartas pararam de se alimentar e se dispersaram procurando um local para a construção do casulo. Neste momento foram individualizadas em recipientes de vidro com capacidade de $500 \mathrm{~mL}$, com papel absorvente umedecido com água destilada, no fundo e tapados com tecido voile branco na extremidade superior. Por transparência do casulo verificou-se a data da formação das pupas.

$\mathrm{Na}$ fase de pupa, no segundo dia após a formação, verificou-se o sexo através da morfologia seguindo-se BuTT \& CANTU (1962); avaliou-se peso, maior largura e comprimento.

$\mathrm{Na}$ fase adulta foram formados 40 casais mantidos em gaiolas cilíndricas de PVC, com diâmetro e altura de $20 \mathrm{~cm}$, revestidas com filme plástico na base e voile branco na porção superior. Estas gaiolas foram forradas, no fundo e nas laterais, com papel filtro, diariamente umedecido com água destilada e também receberam um ramo da planta hospedeira para a oviposição.
Exemplares, em cada fase do desenvolvimento, foram fotografados, fixados e incorporados às coleções do Laboratório de Entomologia, PUCRS e do Laboratório de Biologia, UCS.

Realizou-se um levantamento das plantas hospedeiras citadas em bibliografia e registros de material coletado em campo, atualizando-se a nomenclatura usada por BACKES \& NARDINO (2001), sendo relacionado os nomes vulgares e científicos, famílias e referências.

\section{RESULTADOS E DISCUSSÃO}

O tempo médio de incubação de seis posturas foi de 90,83 $\pm 4,230$ dias. Provavelmente, na temperatura a que foram submetidos neste estudo, ocorreu uma aceleração do desenvolvimento embrionário. Considerando-se que na maior parte das regiões de sua ocorrência as temperaturas médias de inverno apresentam isotérmicas típicas de zonas temperadas, esta espécie, passa o inverno na fase de ovo conforme já referido por DALLAS (1933), Bourquin (1944), CASAlá et al. (1967), Gardiner (1982) LAMPE (1986) e Lemaire (2002). A cobertura das posturas, por cerdas abdominais que servem para impedir ou dificultar a predação, também contribui para uma longa permanência dos ovos no ambiente, como já demonstrado para Hylesia metabus (Cramer, 1775) (Rodrigues et al. 2004). Ainda, segundo Lemaire (2002) várias espécies deste gênero podem passar os períodos desfavoráveis, como estações secas ou frias, em diapausa na forma de ovos.

Os ovos são subcilíndricos, com as extremidades arredondadas, tendo maior largura de 0,816 $\pm 0,005$ e comprimento de $1,103 \pm 0,008(\mathrm{n}=88)$. A coloração é esbranquiçada (Fig. 2) sendo depositados em camadas, recobertos por cerdas de coloração parda, oriundas das regiões latero-posteriores do abdome da fêmea. As posturas (Fig. 3), encontradas sobre os ramos, apresentam base subcircular de 9,7 $\pm 0,42 \mathrm{~mm}$ de diâmetro e $5,4 \pm 0,31 \mathrm{~mm}$ de altura $(\mathrm{n}=10)$, semelhante aos valores relacionados por Bourquin (1944). A deposição das cerdas recobrindo as posturas é característica do gênero (Lemaire 2002) e, como mencionado anteriormente, está relacionada com a proteção contra inimigos naturais (RoDRIGUEs et al. 2004). Quanto à sua importância clínica cabe salientar que, na Argentina, existe a descrição dos componentes das posturas desencadeando processos alérgicos, principalmente em pessoas já sensibilizadas por este agente (Jörg 1933), principalmente pessoas que trabalham podando e limpando as árvores frutíferas, sendo considerada uma enfermidade profissional (CASALÁ et al. 1967).

A viabilidade dos ovos foi de $78,28 \% \pm 5,84$ que pode ser considerada alta em comparação aos $16,2 \% \pm 8,1$ obtidos por SANTOs et al. (1996) para Hylesia nanus (Walker, 1855) criada em condições laboratoriais similares.

As lagartas, ao eclodirem, alimentam-se de imediato do cório e saem do interior da postura através de aberturas circulares (Fig. 4). No exterior andam em fila indiana por dois ou três dias antes de iniciarem a alimentação. De forma similar àquela descrita em LEMAIRE (2002), observou-se uma preferência pelas folhas maduras utilizadas como alimento.

Revista Brasileira de Zoologia 23 (1): 248-255, março 2006 

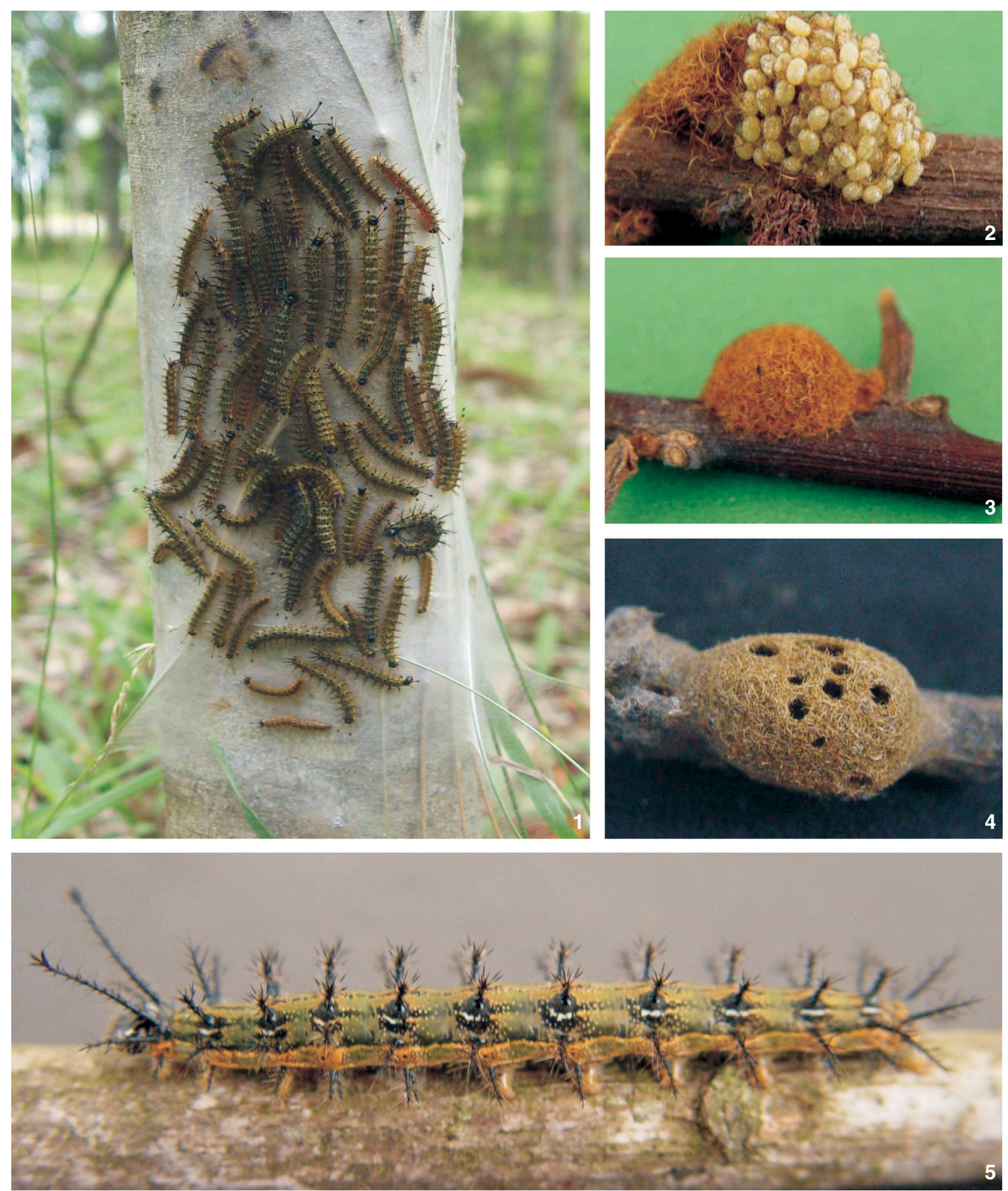

Figuras 1-5. Hylesia nigricans: (1) agregação em campo; (2) postura com ovos expostos; (3) postura; (4) postura com aberturas por onde as lagartas saíram; (5) lagarta de último ínstar.

Revista Brasileira de Zoologia 23 (1): 248-255, março 2006 
As medidas das cápsulas cefálicas e a razão de crescimento entre os instares, expressas na tabela I, foram coerentes com o crescimento previsto pela regra de Dyar (1890).

Detectou-se sete ínstares apenas para as fêmeas. Este ínstar adicional ocorreu devido ao dimorfismo sexual acentuado, onde as fêmeas são muito maiores que os machos, o que também foi observado por GaRdiner (1982) em espécies dos gêneros Dirphia Hübner, [1819] e Periphoba Hübner, [1820] cujo tamanho e peso da fêmea é muito maior que o do macho. Assim, considerandose que o tamanho absoluto das lagartas desencadeia a entrada na fase pupal (Guppy 1969, NiHouth 1975), devido ao seu maior tamanho, as fêmeas necessitam de um instar adicional para atingir o tamanho necessário à sua transformação em crisálida.

O aumento na variação entre as larguras das cápsulas com o desenvolvimento (Tab. I) pode ser atribuído, principalmente, à diferenciação sexual, com fêmeas progressivamente maiores que os machos, especialmente após o quarto instar (ZENNERPolania \& Helgegesen 1973).

Tabela I. Médias da largura da cápsula cefálica com respectivo erro padrão e razão de crescimento, de lagartas de Hylesia nigricans alimentadas com folhas de canela-preta - Nectandra megapotamica, em laboratório.

\begin{tabular}{|c|c|c|c|}
\hline \multirow{2}{*}{ Instar } & \multirow{2}{*}{$\mathrm{N}$} & Largura da cápsula cefálica (mm) & \multirow{2}{*}{$\begin{array}{c}\text { Razão de } \\
\text { Crescimento }\end{array}$} \\
\hline & & $(X \pm E P)$ & \\
\hline 1 & 69 & $0,50 \pm 0,001$ & - \\
\hline II & 140 & $0,72 \pm 0,003$ & 1,44 \\
\hline III & 132 & $1,05 \pm 0,003$ & 1,45 \\
\hline IV & 133 & $1,38 \pm 0,004$ & 1,32 \\
\hline V & 130 & $1,83 \pm 0,006$ & 1,33 \\
\hline VI & 128 & $2,53 \pm 0,015$ & 1,38 \\
\hline VII & 19 & $3,73 \pm 0,095$ & 1,47 \\
\hline
\end{tabular}

A duração da fase larval foi de 67,40 $\pm 1,360$ dias ( $\mathrm{n}=23$ ), com um mínimo de 57 e um máximo de 73 dias. Diversos autores que relatam desenvolvimento desta espécie mencionam a ocorrência das lagartas apenas durante a primavera onde as temperaturas são mais amenas (MABILDE 1896, Bourquin 1944, Casalá et al. 1967, Lampe 1986). Assim, na natureza o período larval deve ser mais prolongado.

Entre os estádios observou-se falta de uniformidade no desenvolvimento das lagartas, tanto das mantidas em laboratório quanto nas monitoradas em campo (Fig. 1), ocorrendo simultaneamente mais de um instar. Este comportamento não era esperado uma vez que para a maioria dos hemileucíneos a agregação leva ao efeito de perfeita sincronia entre as mudas em lagartas provenientes de mesma postura (Lemaire 2002).

O gregarismo acentuado desde o primeiro instar, que não permitiu a criação individual, corrobora as observações de LEMAIRE (2002) e é explicado por fornecer vantagens como termoregulação, redução da perda de água ou aumento da eficiência de forrageamento destacando-se que a interação de dissuadir inimigos naturais é a função mais universal (TulLberg \& HunTER 1996).

Logo após a eclosão até o terceiro instar, as lagartas permaneceram reunidas em locais escuros e abrigadas entre as folhas; após o quarto instar, permaneciam durante o dia em um abrigo e ao anoitecer andavam em fila indiana até as folhas, para alimentarem-se, retornando no decorrer da noite. Tal comportamento é descrito como característico para todas as espécies do gênero (Lemaire 2002). Tanto em laboratório como no campo foi observado o comportamento de andar "em procissão" sendo mais pronunciado nas agregações no campo, onde houve a produção de uma superfície de seda na base das árvores sobre a qual as lagartas permaneceram agrupadas conforme descrito em Bourquin (1944); entre os fios de seda, podem ser observadas ainda as exúvias (Fig. 1). Em laboratório observouse a construção de um "cartucho" onde as lagartas uniram as folhas formando um abrigo em forma de saco como o descrito para H. nanus (SANTOS et al. 1988, 1996).

Com relação ao comportamento de locomoverem-se em fila indiana, ao estudarem o papel dos estímulos químicos e tácteis na formação e manutenção das "procissões" de Hylesia lineata Druce, 1886 já foram discutidos vários aspectos da comunicação química e vantagens adaptativas com relação à procura de novas fontes de alimento, caso ocorra o desfolhamento total da sua arvore hóspede, procura por locais para pupação, e uma exposição aposemática ou intimidadora que pode ter um alto valor para a sobrevivência da espécie frente aos inimigos naturais (Fitzgerald \& Pescador-Rubio 2002).

As lagartas são muito sensíveis a estímulos como sons, movimento, mudanças repentinas de luminosidade ou contato físico. Em algumas ocasiões, principalmente durante a troca de alimento, os indivíduos tocados arqueavam a extremidade anterior do corpo regurgitando um líquido esverdeado, movimentavam-se freneticamente para os lados e depois locomoviam-se rapidamente, contorcendo-se e lançando-se ao fundo da gaiola numa reação comportamental que visa intimidar ou injetar os espinhos no agressor. Somente após uma ou duas horas sem perturbação, estas lagartas voltavam à agregação. Este tipo de reação também foi observado entre os indivíduos das agregações no campo. Reações comportamentais frente a estímulos sonoros e de movimento com respostas semelhantes a estas foram relatados para uma agregação de uma espécie do mesmo gênero na Costa Rica (Hodge 1972). Ainda com relação à sinalização acústica em lagartas YACK et al. (2001) mostram evidências de que é uma forma de comunicação muito difundida entre os lepidópteros, considerada como a principal forma de comunicação larval do grupo.

No primeiro instar as lagartas apresentam coloração amarela com a cápsula cefálica preta, os escolos do pronoto, característicos do gênero, já se apresentam mais desenvolvidos que os demais, com a extremidade dilatada. A cada instar as lagartas tornaram-se mais esverdeadas e escuras, permanecendo com 

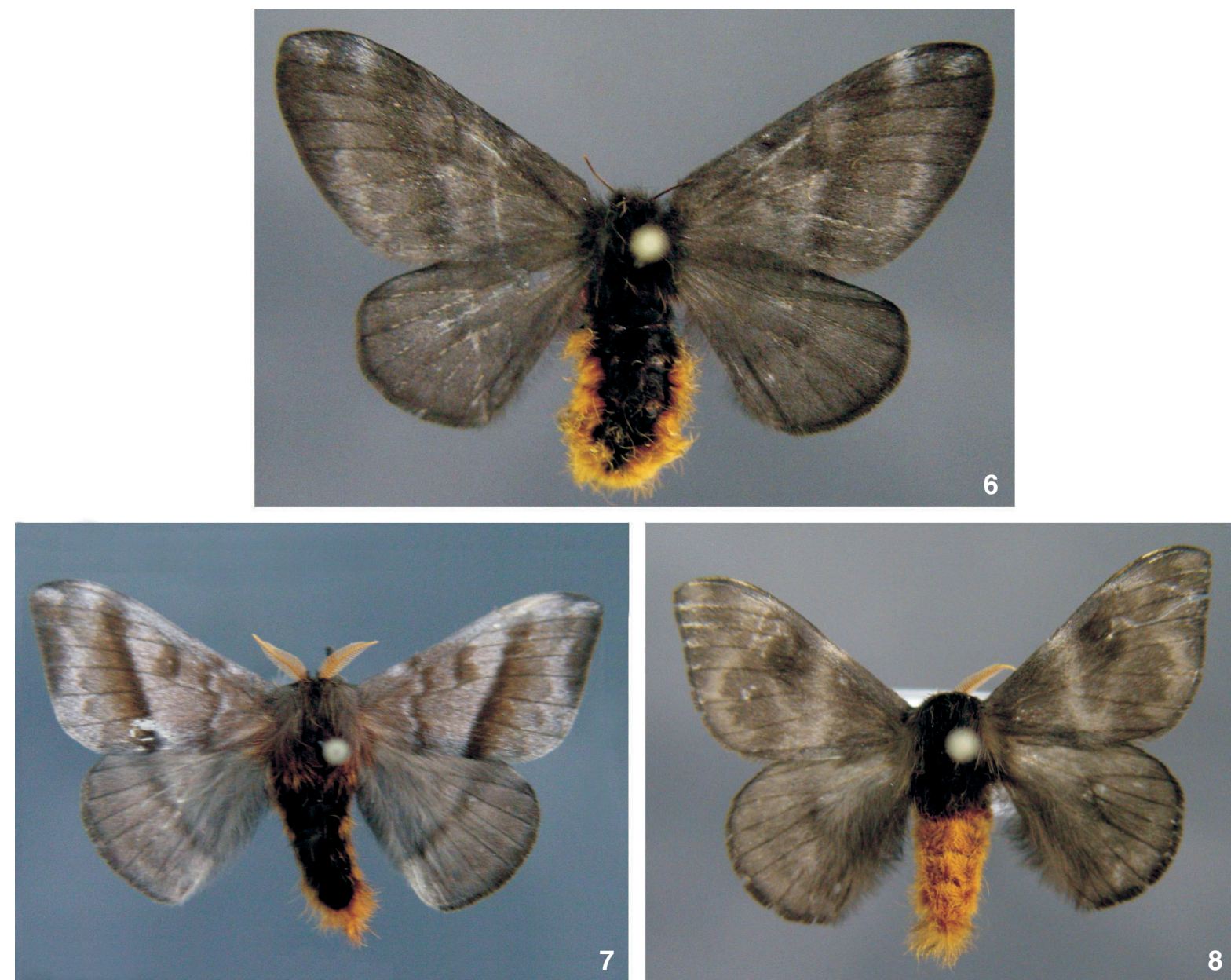

Figuras 6-8. Representantes adultos de Hylesia nigricans: (6) fêmea; (7) macho com abdome de coloração normal; (8) macho com abdome de coloração amarelada.

o mesmo padrão em todos os ínstares. Quando completamente desenvolvidas (Fig. 5) têm comprimento médio de 39,83 \pm $1,225 \mathrm{~mm}(\mathrm{n}=10)$; cabeça de coloração negra brilhante, tegumento preto, densamente ornamentado com pontos brancos; faixa dorsal castanha, lateralmente bordeada com branco; faixa lateral esbranquiçada, bandas espiraculares muito amplas, marrom-esverdeado, a porção inferior bordejada com branco. Escolos pretos, par do pronoto maior com a expansão apical característica do grupo. Espiráculos laranja escuro com borda preta, muito semelhantes à descrição de Lemaire (2002).

A reunião dos dados referentes às plantas hospedeiras permitiu relacionar plantas pertencentes a 14 famílias (Tab. II), destacando-se os representantes de Rosaceae, Salicaceae e Lauraceae. O registro da diversidade de hospedeiros indica uma grande facilidade de adaptação a diferentes grupos vegetais relacionando-se esta espécie como polifitófaga a exemplo de outras mais estudadas como Hylesia lineata Druce, 1886 (JANSEN 1984).
O período de pré-pupa foi caracterizado pela parada da atividade de alimentação e abandono do abrigo em procura de um lugar para a confecção do casulo, observando-se uma duração de 4,94 $\pm 0,206$ dias $(\mathrm{n}=21)$.

Na fase de pupa observou-se que as fêmeas $(n=38)$ foram significativamente maiores que os machos $(\mathrm{n}=38)$ com massa de 0,50 $\pm 0,009$ e 0,31 $\pm 0,004$ g; comprimento de 18,36 $\pm 0,174 \mathrm{~mm}$ e $15,37 \pm 0,114 \mathrm{~mm}$; largura de $6,83 \pm 0,053 \mathrm{~mm}$ e $6,06 \pm 0,047 \mathrm{~mm}$, respectivamente. Este comportamento era esperado em função do grande dimorfismo sexual característico do grupo, também relatado para esta espécie (Lemaire 2002). Apesar da grande variação entre indivíduos, não se observou diferença significativa para a duração deste período entre os sexos que foi de $35,92 \pm 2,576$ dias $(\mathrm{n}=19)$, bastante semelhante à observada por BourQuin (1944) e LAMPE (1986), diferindo de Mabilde (1896) que relaciona em torno de 80 a 90 dias.

Na fase adulta observou-se que as fêmeas (Fig. 6) apresen-

Revista Brasileira de Zoologia 23 (1): 248-255, março 2006 
Tabela II. Plantas hospedeiras de Hylesia nigricans segundo vários autores e dados obtidos durante o presente estudo.

\begin{tabular}{|c|c|c|c|}
\hline Nome vulgar & Nome científico & Família & Referência \\
\hline Acácia & Acacia sp. & Mimosaceae & 6 \\
\hline Álamo & Populus nigra Linnaeus & Salicaceae & 6 \\
\hline Álamo-da-carolina & Populus deltoides Marshall & Salicaceae & 6 \\
\hline Álamo-preto & Populus nigra italica (Moench) Koehne & Salicaceae & 6 \\
\hline Álamo-branco & Populus alba Linnaeus. var. pyramidalis Bungue & Salicaceae & 4 \\
\hline Álamos & Populus spp. & Salicaceae & $2,3,6$ \\
\hline Ameixeira & Prunus domestica Linnaeus & Rosaceae & $4,5,6$ \\
\hline Amendoeira & Prunus amygdalus Batsch & Rosaceae & 6 \\
\hline Canela & Ocotea acutifolia (Ness.) Mez. & Lauraceae & 6 \\
\hline Canela-canfora & Camphoromoea laxa Ness & Lauraceae & 2 \\
\hline Canela-do-brejo & Ocotea pulchella Mart. & Lauraceae & 6 \\
\hline Canela-preta & Nectandra megapotamica (Spreng.) Mez & Lauraceae & * \\
\hline Carpino & Carpinus betulus Linnaeus & Betulaceae & 8 \\
\hline Cerejeira-europeia & Prunus avium Linnaeus & Rosaceae & 6 \\
\hline Carvalho-europeu & Quercus robur Linnaeus & Fagaceae & 6 \\
\hline Chorão & Salix babylonica Linnaeus & Salicaceae & 4,6 \\
\hline Damasqueiro & Prunus armeniaca Linnaeus & Rosaceae & 6 \\
\hline Erva-mate & Ilex paraguariensis St. Hil. & Aquifoliaceae & 3,7 \\
\hline Eucalipto & Eucaliptus sp. & Myrtaceae & 9 \\
\hline Guabirobeira & Campomanesia xanthocarpa Berg & Myrtaceae & $1,5,6$ \\
\hline Guajuvira & Patagonula americana Linnaeus & Boraginaceae & 6 \\
\hline Macieira & Pyrus malus Linnaeus & Rosaceae & 4 \\
\hline Macieira-brava & Malus sylvestris Mill. & Rosaceae & 6 \\
\hline Macieiras & Malus sp. & Rosaceae & 8 \\
\hline Marmeleiro & Cydonia oblonga Mill. & Rosaceae & 6 \\
\hline Nogueira-pecan & Carya illinoinensis K. Koch & Juglandaceae & * \\
\hline Pessegueiro & Prunus persica (Linnaeus) Stokes & Rosaceae & $4,6,7$ \\
\hline Pereira & Pyrus comunis Linnaeus & Rosaceae & 6 \\
\hline Plátano & Platanus spp. & Platanaceae & 9 \\
\hline Plátano-falso & Acer sp. & Aceraceae & 9 \\
\hline Quaresmeira-da-serra & Tibouchina sellowiana Cogn. & Melastomataceae & * \\
\hline Salgueiro & Salix humboldtiana Wild & Salicaceae & 4,6 \\
\hline Salgueiros & Salix spp. & Salicaceae & 2,9 \\
\hline Tipuana & Tipuana tipu (Benth.) Kuntze & Cesalpiniaceae & * \\
\hline
\end{tabular}

* Observações do presente estudo, 1) Mabilde (1896), 2) Draudt (1929), 3) Bourquin (1944), 4) Biezanko et al. (1957), 5) Silva et al. (1968), 6) Biezanko et al. (1974), 7) Biezanko $(1986), 8)$ Lampe $(1986), 9)$ Latorre (2003).

taram pouca variação na coloração, porém entre os machos foram acentuadas, principalmente quanto à coloração das asas e abdome. Observaram-se duas formas: uma cuja coloração das asas apresenta desenhos bastante nítidos, com abdome de coloração escura (Fig. 7) e outra com coloração das asas pouco definida e abdome amarelado (Fig. 8). Esta última forma foi observada em pelo menos $20 \%$ dos machos que emergiram em laboratório. Segundo Lemaire (2002) a identificação específica das espécies de
Hylesia é muito difícil devido às semelhanças de seus representantes. Este estudo acrescenta ainda, variação intraespecífica, que também pode ocorrer em outros representantes deste gênero.

A envergadura das fêmeas $(n=38)$ foi significativamente maior com tamanho médio $52,40 \pm 0,359$ mm enquanto os machos $(\mathrm{n}=38$ ) apresentaram $38,64 \pm 0,489 \mathrm{~mm}$. Estes resultados revelam adultos com envergadura semelhante àquela relatada por Lemaire (2002). Estes resultados aliados à manutenção de 
alguns exemplares até a quarta geração, indicam que as condições de laboratório supriram satisfatoriamente as exigências desta espécie.

Observou-se uma média de 1,13 $\pm 0,063$ posturas $(\mathrm{n}=$ 30); o número médio de ovos por fêmea foi de $285,83 \pm 26,355$ ( $\mathrm{n}=12$ ) com um mínimo de 138 e máximo de 511 . Estes resultados diferem das observações de Bourquin (1944) que registra a ocorrência de aproximadamente 900 ovos.

Após a oviposição não foi constatada a presença de ovos no interior do abdome das fêmeas estudadas conforme descrito para H. nanus (SANTos et al. 1988, 1996), porém o número de ovos foi bastante semelhante ao encontrado por aqueles autores quando computados os das posturas mais os retidos no abdome $(297,3)$; da mesma forma quando comparados com os de H. lineata que oviposita em torno de 270 ovos (Fitzgerald \& Pescador-Rubio 2002).

A longevidade média foi de 6,09 $\pm 0,142$ dias $(n=72)$; os machos foram mais longevos que as fêmeas com 6,33 $\pm 0,273$ $(\mathrm{n}=42)$ e $5,33 \pm 0,168(\mathrm{n}=30)$ dias, respectivamente. A longevidade dos machos foi muito semelhante da observada para $H$. nanus (SANTos et al. 1996) porém as fêmeas viveram um dia a menos. Os períodos médios de pré, pós e oviposição foram 3,17 $\pm 0,196 ; 1,14 \pm 0,064$ e $1,10 \pm 0,057$ dias, respectivamente. É característico dos hemileucíneos não se alimentarem durante a fase adulta, necessitando a lagarta armazenar toda a energia para as fases seguintes (Lemaire 2002). Presume-se que a curta longevidade, com tempo de pré-oviposição relativamente mais extenso, esteja relacionado com as atividades de atração e localização do sexo oposto, cópula e localização da planta hospedeira para que finalmente seja realizada a oviposição, finalizando o ciclo.

Os adultos ocorrem entre os meses de novembro a maio (BIEZANKo et al. 1957, BIEZANKo 1986) causando dermatites sempre entre o final da primavera e o verão. Acredita-se que, em função de variações climáticas como as desencadeadoras do fenômeno El Niño e de La Niña, caracterizados por invernos mais amenos ou mais rigorosos, respectivamente (LASMAR 1997), podem ocorrer antecipações ou retardamentos no ciclo vital desta espécie, o que explicaria as diferenças encontradas para a grande variação entre as épocas de emergência dos adultos.

Nas condições em que foi realizado o presente estudo, observou-se que o ciclo de vida desta espécie foi de aproximadamente 206 dias, porém na natureza, especialmente nas fases de ovo e de lagarta, em função da temperatura, o tempo necessário para o desenvolvimento deve ser muito maior, uma vez que se trata de uma espécie univoltina que passa inverno na fase de ovo (Dallas 1933, Bourquin 1944, Casalá et al. 1967, Gardiner 1982, Lampe 1986, Lemaire 2002).

Apesar de serem referidos os parasitóides Coccygominus tomyris Schrottky, 1902 (Hymenoptera, Ichneumonidae); Telenomus almanzori Marelli, 1937; Telenomus hyelosiae (Brèthes, 1909); Telenomus sp. (Hymenoptera, Scelionidae) e Belvosia leucopyga van der Wulp, 1882 (Diptera, Tachinidae) (Peigler
1994), durante este trabalho, entre os materiais estudados, não foi constada presença de inimigos naturais.

\section{AGRADECIMENTOS}

À FAPERGS, pela concessão da Bolsa de Iniciação Científica (Proc. $n^{\circ}$ 02/508357) e pelo Auxílio Financeiro (Proc. $n^{\circ}$ 02/1739.6). À Dra. Liane Terezinha Dorneles, pela coleta de diversas posturas em campo; ao Doutor Josué Sant'Ana, pela tradução do resumo e doutores Rosane Maria Lanzer e Alois Eduard Schäfer, pela tradução da bibliografia na língua alemã.

\section{REFERÊNCIAS BIBLIOGRÁFICAS}

Arias, H. 1981. Dermatitis por Hylesia nigricans. Archivos Argentinos de Dermatología, Buenos Aires, 31 (3): 171-180.

BACKes, A. \& M. NARdino. 2001. Nomes populares e científicos de plantas do Rio Grande do Sul. São Leopoldo, Unisinos, Coleções Fisionomia Gaúcha, 2a ed. 202p.

BERG, C. 1875. Lepidópteros Patagónicos observados en el viaje de 1874. Actas de la Academia Nacional de Ciencias de la Universidad de Cordoba, Cordoba, 1: 63-102, 155-158.

Biezanko, C.M. 1986. Adelocephalidae, Saturniidae, Mimallonidae, Lasiocampidae, Eupterotidae e Lymantriidae da Região missioneira do Rio Grande do Sul. Revista do Centro de Ciências Rurais, Santa Maria, 16 (2): 89-112.

Biezanko, C.M.; A. Ruffinelli \& C.S. Carbonell. 1957. Lepidoptera del Uruguay - lista anotada de espécies. Revista de la Faculdad de Agronomía, Montevideo, 46: 1-152.

Biezanko, C.M.; A. Ruffinelli \& D. Link. 1974. Plantas y otras sustâncias alimentícias de las orugas de los lepidópteros uruguayos. Revista do Centro de Ciências Rurais, Santa Maria, 4 (2): 107-148.

Biezanko, C.M.; A. Ruffinelui \& D. Link. 1978. Catálogo de lepidópteros do Uruguai. Revista do Centro de Ciências Rurais, Santa Maria, 8 (Supl.): 1-84.

Bourquin, F. 1944. XII - Observaciones sobre Hylesia nigricans (Berg) 1875, p. 45-47. In: F. BouRquin (ED.). Mariposas argentinas - vida desarrollo, costumbres y hechos curiosos de algunos lepidópteros argentinos. Buenos Aires, El Ateneo, 213p.

Butt, B.A. \& E. CANTU. 1962. Sex determination of lepidopterous pupae. Washington. USDA, 7p.

Casalá, A.; C. Bianchi; J.V.S. Navarro; O. Bianchi \& R. Balsa. 1967. Granulona de lãs manos por nidos de lepidópteros (Hylesia nigricans). Archivos Argentinos de Dermatologia, Buenos Aires, 17 (4): 307-314.

Corseuil, E.; A. Specht \& C. Lang. 2002. Saturniídeos (Lepidoptera, Saturniidae) registrados para o Rio Grande do Sul, Brasil. I. Hemileucinae. Biociências, Porto Alegre, 10 (2): 147-155.

Dallas, E.D. 1926. Eritema producido por un lepidóptero. Revista de la Sociedad Entomológica Argentina, Buenos Aires, 1 (2): 63-64.

Revista Brasileira de Zoologia 23 (1): 248-255, março 2006 
Dallas, E.D. 1933. 16 - Outro caso de dermitis extendida, producida por um lepidóptero y notas sobre Hylesia nigricans Berg (Lep. Bombicidae), p. 469-474. In: Anais $8^{\text {a }}$ Reunion de la Sociedad Argentina de Patologia Regional del Norte. Buenos Aires, Universidade de Buenos Aires, 1063p.

Draudt, M. 1929. 12 - Familie Saturniidae, p. 713-827. In: A. SeItZ (Ed.). Die Gross-Schmetterlinge der Erde. 6. Die Amerikanischen Spinner und Schwärmer. Stuttgart, Alfred Kernen, 508p.

Dyar, H.G. 1890. The number of molts of lepidopterous larvae. Psyche, Cambridge, 5: 420-422.

Fitzgerald, T.D. \& A. Pescador-Rubio. 2002. The role of tactile and chemical stimuli in the formation and maintenance of the processions of the social caterpillar Hylesia lineata (Lepidoptera: Saturniidae). Journal of Insect Behavior, New York, 15 (5): 659-674.

Gardiner, B.O.C. 1982. A Silkmoth Rearer's Handbook. Hanworth, The Amateur Entomological Society, $3^{\text {rd }}$ ed., 255p.

Guppy, J.C. 1969. Some effects of temperature on the immature stages of the armyworm, Pseudaletia unipuncta (Lepidoptera: Noctuidae), under controlled condictions. The Canadian Entomologist, Ottawa, 101: 1320-1327.

Haddad, V. \& J.L.C. Cardoso. 2003. 22 - Erucismo e Lepidopterismo, p. 220-223. In: J.L.C. Cardoso; F.O.S. FranÇA; F.H. Wen; C.M.S. MÁlaque \& V. HADDAD (Eds). Animais peçonhentos no Brasil - biologia, clínica e terapêutica dos acidentes. São Paulo, Sarvier, 468p.

Hodge, C.L. 1972. Protective function of sound percepetion and gregariousness in Hylesia larvae (Sturniindae: Hemileucinae). Journal of the Lepidopterist' Society, Los Angeles, 26 (1): 33-34.

JANSEN, D.H. 1984. Natural history of Hylesia lineata (Saturniidae: Hemileucinae) in Santa Rosa National Park, Costa Rica. Journal of Kansas Entomological Society, Manhattan, 42 (1): 132-137.

JÖRG, M.E. 1933. Nota previa sobre el princípio activo urticante de Hylesia nigricans (Lepidopt. Hemileucinae) y lãs dermitis provocadas por el mismo, p. 482-495. In: Anais $8^{\text {a }}$ Reunion de la Sociedad Argentina de Patologia Regional del Norte. Buenos Aires, Universidade de Buenos Aires, 1063p.

JörG, M.E. 1935. Dermatosis lepidopterianas (segunda nota), p. 1617-1639. In: Anais 9a Reunion de la Sociedad Argentina de Patologia Regional del Norte. Buenos Aires, Universidade de Buenos Aires, vol. 3. [1939]

JöRG, M.E. 1969. Subcutaneous inflammatory nodule caused by spicules of Hylesia fulviventris (Lepidoptera). Boletin Chileno de Parasitologia, Santiago, 24 (3): 146-150.

LAMPE, R.E.J. 1986. Die Präimaginalstadien von Hylesia nigricans Berg, 1875 (Lep.: Saturniidae). Entomologische Zeitschrift, Frankfurt, 96: 273-277.

LASMAR, A. 1997. A fúria da natureza. Ecologia e Desenvolvi- mento, Rio de Janeiro, 7 (1): 16-19.

Latorre, E.M. 2003. Plagas Florestales. Disponível na World Wide Web em: http://www.ilustrados.com/publicaciones/ EpykZZEVFpxYDtDPug.php [acesso em 20/II/2006]

Lemaire, C. 2002. The Saturniidae of America - Hemileucinae. Keltern, Goecke \& Evers, vol. 3, 1388p.

Mabilde, A.P. 1896. Guia práctico para os principiantes collecionadores de insectos, contendo a descrição fiel de perto de 1000 borboletas com 180 figuras lytographadas em tamanho, formas e desenhos conforme o natural. Estudo sobre a vida de insectos do Rio Grande do Sul e sobre a caça, classificação e conservação de uma coleção mais ou menos regular. Porto Alegre, Gundlach \& Schuldt, 238p.

Niноuтh, H.F. 1975. A threshold size for metamorphosis in the tobacco hornworm, Manduca sexta (L.). The Biological Bulletin, Woods Hole, 149: 214-225.

PARRA, J.R.P. \& M.L. HADDAD. 1989. Determinação do número de ínstares de insetos. Piracicaba, FEALQ, 49p.

PeIgler, R.S. 1994. Catalog of parasitoids of Saturniidae of the World. Journal of Research on the Lepidoptera, Beverly Hills, 33: 1-121.

Rodriguez, J.; J.V. Hernandéz; L. Fornés; U. Lundberg; C.L. ArochaPIÑANGo \& F. Osborn. 2004. External morphology of abdominal setae from male and female Hylesia metabus adults (Lepidoptera: Saturniidae) and their function. Florida Entomologist, Gainesville, 87 (1): 30-36.

Santos, G.P.; N. Anjos \& J.C. Zanuncio. 1988. Biologia de Hylesia nanus (Walker, 1855) (Lepidoptera: Attacidae), desfolhadora da cutieira (Joannesia princeps: Euphorbiaceae). Revista Ceres, Viçosa, 35 (201): 479-485.

SAntos, G.P.; T.V. Zanuncio; O.S. Dias \& J.C. Zanuncio. 1996. Biologia de Hylesia nanus (Walker) (Lepidoptera: Attacidae). Anais da Sociedade Entomológica do Brasil, Londrina, 25 (3): 479-482.

Silva, A.G.A.; C.R. Gonçalves; D.M. Galvão; A.J.L. Gonçalves; J. Gomes; M.M. Silva \& L. Simoni. 1968. Quarto catálogo dos insetos que vivem nas plantas do Brasil: seus parasitos e predadores. Rio de Janeiro, Ministério da Agricultura, 622p.

Tullberg, B.S. \& A.F. HunTER. 1996. Evolution of larval gregariousness in relation to repellent defences and warning coloration in tree-feeding Macrolepidoptera: a phylogenetic analysis based on independent contrasts. Biological Journal of the linnean Society, London, 57: 253-276.

YACK, J.E; M.L. SMith \& P.J. Weatherhead. 2001. Caterpillar talk: acoustically mediated territoriality in larval lepidoptera. Proceedings of the National Academy of Science, Washington, 98 (20): 11371-11375.

Zenner-Polania, I.; R.G. Helgegesen. 1973. Effect of temperature on instar number and head-capsule width of Platynota stultana (Lepidoptera: Tortricidae). Environmental Entomology, College Park, 2: 823-827.

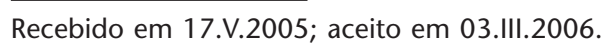

Revista Brasileira de Zoologia 23 (1): 248-255, março 2006 